Cerebrovascular
Diseases

\title{
$14^{\text {th }}$ ESNCH Meeting, Riga, Latvia, 2009
}

\section{Congress Lectures}

In chronological order

\section{SUNDAY, 24TH MAY 2009}

\section{SESSION I}

\section{Carotid plaque angiogenesis detection with contrast ultrasound}

E. Vicenzini

Department of Neurological Sciences

Sapienza, University of Rome, Rome, Italy

Background: Histological studies have recognized that the presence of adventitial vasa vasorum and intra atheroma neoangiogenesis are predictors of plaques instability. Contrast carotid ultrasound has proven a reliable tool to identify "in vivo" these features. This technique and its clinical implications are discussed.

Materials and Methods: Standard Duplex and Contrast Ultrasound have been performed both in symptomatic and asymptomatic plaques of different morphology and degree of stenosis. In operated patients, histology was used to confirm the patterns of vascularization observed at contrast ultrasound.

Results:Vascularization was detected at the plaque shoulders, in the fibrous and in the fibrofatty tissues. A small vessel was constantly observed below ulcerations. The diffusion of microbubbles was from adventitial layers towards the inside of plaques. Vascularization was not detected in calcifications or in hemorrhagic areas. Acute symptomatic plaques showed a different vascularization pattern in respect to asymptomatic ones, with a more diffuse contrast enhancement in localized areas of the lesions.

Conclusions: Contrast Ultrasound depicts different vascularization patterns in carotid plaques. In symptomatic and asymptomatic patients with surgical indications, contrast examinations may add "in vivo" data on the evaluation of cerebrovascular risk, identifying more "unstable" plaques with a higher degree of vascularization. Future studies will clarify the prognostic value of neoangiogenesis in plaques of asymptomatic patients with non-severe, non-surgical carotid stenosis. Finally, possible effects of therapies aimed to plaque remodelling with effects on neoangiogenesis may be monitored with contrast ultrasound.

\section{Detection of rupture-prone atherosclerotic plaques Holger.Poppert Neurological Clinic of Technical University of Munich Münich-Germany}

In contrast to "lumenographic" methods (digital subtraction angiography, CT angiography, time-of-fight and phase contrast MR angiography), B-mode ultrasound enables the examiner to directly image walls of arteries and atherosclerotic plaques. Echogenicity and echo texture have been reported to correlate with plaque composition and potential disposition to rupture, although histological evaluation showed only poor concordance in some studies. Surface irregularities and inhomogeneity of the plaque have been proposed as features of unstable plaques, though this is difficult to quantify objectively. A number of longitudinal studies have investigated the correlation of these sonographic features in patients with carotid atheroma and incidence rate of stroke with promising but conflicting results. Although B-mode ultrasound has considerable potential for detection of vulnerable plaques, it is currently limited by the fact that results are highly observer dependent. Techniques to overcome this limitation by improving B-mode image quality and development of dedicated software for image normalization and interpretation are under investigation. A further promising approach is visualization of intraplaque neovascularization by micro bubble-based ultrasound contrast medium.

Like B-mode ultrasound, MRI using a multi-sequence imaging protocol allows direct imaging of the walls of arteries, with the additional advantage of precisely quantifying wall dimensions, plaque composition, and stenosis in a way that is broadly observer independent.

Both methods for non-invasive assessment of plaque morphology will be reviewed.

\section{SESSION II}

\section{Vascular cognitive impairment \\ Stefan Knecht \\ Dept. of Neurology, University of Münster and Neurocenter Schön Klinik Hamburg}

Dementia can be conceptualized as the final common pathway of diseases that disintegrate information processing in the brain. The underlying causes may differ (degenerative, metabolic, vascular, or inflammatory processes) and act simultaneously as well as cumulatively. Because dementia develops towards the end of life, delaying the development of dementia by 2 years might reduce the overall prevalence of dementia by $25 \%$. The pathogenetic factors contributing to brain disintegration that can be treated best are metabolic

Cerebovasc Dis 2009;27 (suppl 5): 1-39 
and vascular disease. However, to reduce accumulation of brain damage over time, interventions have to start early. This makes vascular cognitive impairment a critical concept. Here I report on the SEARCH Health study, a cohort study of 500 community-dwelling individual assessed for the contribution of metabolic and vascular risks to cognition. Data demonstrate that treatable metabolic and vascular conditions account for at least $20 \%$ of variance in cognitive function and presumably risk for dementia.

\section{Is Vascular cognitive impairment a useful concept? Bornstein N.M. Department of neurology, Tel-Aviv Sourasky Medical Center Tel-Aviv, Israel}

Epidemiological data have demonstrated that risk factors for vascular disease in general, and for stroke in particular, are largely identical to those of Alzheimer's disease (AD). Thus, hypertension, diabetes mellitus and elevated serum levels of cholesterol and homocysteine contribute to the occurrence of both diseases. The brain of elderly people accumulates amyloid, neurofibrillary tangles, as well as vascular lesions, large and small, cortical and deep. Blood vessels may manifest lipohyalinosis and result in leucoaraiosis. Cognitive decline in old age results from this cumulative burden. The clinical distinction between vascular dementia and $A D$ is clinically impossible in most cases, is confusing and counterproductive. Most elderly patients with dementia do not have a monolithic pathologic disorder (e.g. AD or vascular dementia) but rather a combination of both. This overlap is supported by imaging evidence as well as by neurochemical markers. Unfortunately, mixed dementia, the most common cause of dementia, is not yet defined clinically or pathologically. Public health measures should be taken to lower the impact of the risk factors responsible for these diseases (such as hypertension, hyperlipidemia, hyperhomocysteinemia, and smoking). Attention to these risk factors by drugs and lifestyle is important in reducing the incidence and prevalence of dementia.

\section{SESSION III}

\section{Progress in Clinical Sonothrombolysis}

Andrei V. Alexandrov, MD

Comprehensive Stroke Center, University of Alabama Hospital, Birmingham, AL, USA

Systemic tissue plasminogen activator (tPA) remains the fastest way to initiate treatment for acute ischemic stroke. The presence of a proximal arterial occlusion should not be viewed as an un-surmountable predictor of tPA failure, and early augmentation of fibrinolysis to improve recanalization can be safely achieved at bedside with diagnostic Doppler ultrasound. In CLOTBUST trial, 83\% of patients achieved any recanalization (46\% complete, $27 \%$ partial) with tPA+ transcranial Doppler vs 50\% (17\% complete, $33 \%$ partial) with tPA alone within 2 hours of treatment, $p<0.001$. Sustained complete recanalization at 2 hours was $38 \%$ vs $13 \%$ respectively, $p=0.03$. A recent pooled analysis of 6 randomized and 3 non-randomized clinical studies of sonothrombolysis showed that any diagnostic ultrasound monitoring can at least double the chance of early complete arterial recanalization at no increase in the risk of symptomatic intracerebral hemorrhage. Since human application of frequencies below diagnostic range resulted in increased symptomatic bleeding rates, mechanisms by which megahertz and kilohertz frequencies interact with clot-residual flow interface and endothelium are currently under renewed investigations.

Catheter-based ultrasound delivery to arterial thrombi and intra-ventricular clots is subject of ongoing clinical trials. Addition of gaseous perflutren-lipid micro spheres to tPA and transcranial Doppler can further facilitate early flow improvement with $>50 \%$ rate of early complete recanalization and the recently completed multi-center international TUCSON trial showed a safe dose of microspheres that can be combined with systemic t-PA. With support from the SPOTRIAS program grant (NIH-NINDS), transcranial ultrasound delivery in an operator-independent and dose-controlled manner is being tested in a clinical trial.

The first International Sonothrombolysis Conference impressions and future directions.

S. Meairs

(Mannheim-Germany)

Abstract not received

\section{SESSION IV}

Imaging of cerebral vasculature and vascularisation neuroradiologist's point of view

S.Dzelzite

(Riga-Latvia)

Abstract not received

$14^{\text {th }}$ ESNCH Meeting, Riga, Latvia, 2009 


\author{
Advances in real-time brain perfusion imaging \\ Stephen Meairs, MD, PhD \\ Department of Neurology, Mannheim \\ University of Heidelberg
}

In the last several years great progress has been made in the field of ultrasound perfusion imaging of the brain. Different approaches have been assessed and shown to be capable of early detection of cerebral perfusion deficits. Real-time low mechanical index imaging simplifies the acquisition of perfusion parameters and offers important advantages over previous ultrasound techniques for evaluation of brain tissue perfusion. First, this approach avoids the shadowing effect, a significant problem associated with high mechanical index imaging. Because of the high acoustic intensities that are emitted by bursting bubbles when high mechanical index imaging is applied, bubbles that are distal to the emitting bubbles are "shadowed" by this effect and thus obscured from data analysis. Areas of tissue that are shadowed may not be available for analysis of tissue perfusion. The problem of shadowing is eliminated with low mechanical index imaging, since bubbles are not destroyed with such low acoustic pressure. Secondly, low MI real-time brain imaging allows acquisition of multi-planar perfusion images. This is a tremendous breakthrough for ultrasound perfusion imaging, since previous approaches were confined to a single image plane and therefore limited in their assessment of the extents of brain infarction and low perfusion states. A further advantage is that the technique can provide exact time-to peak calculations of contrast bolus arrival, thus providing better time resolution for bolus kinetics. Importantly, bubbles are not destroyed with low mechanical index imaging. This opens new possibilities for the development of quantitative perfusion analysis using refill kinetics. Finally, real time imaging enables tracking of micro bubbles within the brain microcirculation allowing for novel post-processing image assessment and parametric imaging.

One limitation of low MI contrast imaging is the very low signal levels returned from bubbles at low acoustic pressures. Sensitivity can be increased by transmitting longer pulses, but at the expense of poorer axial resolution. Coded contrast harmonics $(\mathrm{CCH})$ offers a solution to this dilemma. Pulse compression methods may be combined with the nonlinear bubble imaging techniques for highly sensitive contrast imaging with very low noise. One of the most striking aspects of $\mathrm{CCH}$ is that the contrast lasts much longer than with conventional imaging methods. Thus, with the advent of highly sensitive contrast-specific imaging techniques, new possibilities of real-time assessment of brain infarctions and cerebral hemorrhages have emerged.
MONDAY, 25TH MAY 2009

\section{SESSION V}

\author{
TCD for diagnosing and monitoring of intracranial \\ atherosclerosis \\ L. Wong \\ (Hong-Kong, China) \\ Abstract not received
}

\section{Endovascular interventions \\ G .E.Klein}

(Graz-Austria)

Abstract not received

\section{SESSION VI}

\section{Cerebral monitoring using Transcranial Doppler \\ D.Russell \\ Department of Neurology, Oslo University Hospital \\ Oslo, Norway}

Transcranial Doppler (TCD) is the only method which provides beat-to-beat information regarding both blood flow and embolization in the major intracranial arteries. It is therefore an excellent method for monitoring the cerebral circulation during invasive cardiovascular examinations and cardiovascular operations.

Emboli may be detected since they cause an increase in the amount of reflected ultrasound compared to that normally caused by red blood cells. Mean blood flow velocities provide information regarding changes in blood flow and this method will hopefully be improved by the introduction of frequency-weighted Doppler power measurements.

A major risk during carotid endarterectomy and stenting is ipsilateral ischemic brain injury. It has been shown that solid and gaseous microemboli during carotid endarterectomy and stenting are increased in patients with procedurerelated ipsilateral ischemic strokes or new diffusion-weighted cerebral MRI (DWI) lesions. This suggests that both solid and gaseous emboli may be harmful to the brain during these procedures.

Coronary artery bypass grafting (CABG) is the most common major surgical procedure. Gaseous and solid microemboli, detected using TCD, are frequent during CABG especially during manual manipulation, cannulation and clamping of the ascending aorta.DWI studies have shown new ischemic lesions in $26-45 \%$ of patients after surgery Off-pump CABG surgery significantly decreases the number of emboli entering the brain but this may in part be counteracted by 
more unstable cerebral hemodynamics.

TCD monitoring has also shown that cerebral morphological changes demonstrated with DWI and acute cognitive impairment following left heart catheterization are most likely due to cerebral microembolism, especially solid microemboli.

\section{Orbital ultrasound to monitor the head and retrobulbar optic nerve}

M.Siebler

Essen, Germany

The ophthalmic inspection of the ocular fundus by a neurologist is one of the neurological basic examinations especially if a raised intracranial brain pressure is assumed. The optic disc swelling (ODS, protrusion papillae) as well as the swelling of the proximal optic nerve is associated with increased brain tissue pressure. Hence, optic nerve sonography may serve as an additional diagnostic tool that could alert clinicians to the presence of elevated ICP, whenever invasive ICP evaluation is contraindicated and/ or is not available. The problem is also that neither an ODS is present in all kind of raised brain pressures neither a normal ocular fundus exclude increased brain pressure. Thus, it is a permanent debate whether in all cases of lumbar puncture ODS should be excluded. Therefore, some authors recommended a cerebral brain scan before each lumbar punction.

The clinical ophthalmic investigation especially in emergency situations is not very easy and thus the diagnostic reliability in respect to quality (ODS present or not) and quantification of ODS is uncertain. The funduscopy by an eye specialist is not common practice and cost effective and on ICU restricted. In addition, there is no information about the proximal optic nerve or the optical nerve sheet diameter (ONSD) by funduscopy.

Meanwhile, ultrasound machines with B-images and with a high technical quality standard are present in all departments (internal, intensive care units, neurology). It was Rune Aaslid who had introduced the transorbital insonation with a $2 \mathrm{MHz}$ probe to insonate the distal part of the internal carotid artery (1). However, the insonation power as well as the technical security of the machines was rather minor controlled at this time. Thus, ophthalmologists were be afraid by the possible mechanical and terminal effect of ultrasound on the eye especially the vitreous body as well as on the retina. The new generations of ultrasound machines however had very good control about the emission of radiation power despite an improved image quality with a high time - (ms) and space resolution $(<0.1 \mathrm{~mm})$.

With a conventional ultrasound planar duplex probe (7-12
$\mathrm{MHz}$ insonation frequency) the transpalpetral insonation of the orbita is very easy to perform and the fundus as well as the proximal optic nerve can be displayed, stored and anatomical situation could be measured. The display of the nerve as well as of the papilla is given within 10-20 seconds and could be documented directly (Fig 1 ). We found normal values for the papilla were $<0.6 \mathrm{~mm}$ (Fig 2). The method is excellent to control the development and effectiveness of therapeutic interventions (Fig 3). The handling is easy to learn even for non-specialists.

ONSD measurements correlate with non-invasive and invasive measurements of the ICP, and with head computed tomography scan findings in brain-injured adults. In the same session, the display of the proximal optic nerve is also available (Fig 2; 3). The normal optic nerve of adults is in the range $<6 \mathrm{~mm}$. We observed increased ONSD in patients with increased intracranial pressure before ODS which is compatible to observation from Soldatos et al.

If the duplex mode was used the perfusion of the optic disk as well as the blood flow velocity within the central retinal artery could be measured (example will be given in the presentation).

To avoid damages each ultrasound machine should be controlled whether the limits on ultrasound radiation is given. The following parameters are relevant and FDA approved: MI: $<0.23 ; \mathrm{Tl}<0.2 ;$ Ispta $<17 \mathrm{~mW} / \mathrm{cm}^{2}$. We recommend installing a special programme on the machine for the investigation.

Conclusion: Orbita ultrasound imaging is easy to perform and give relevant information about ODS as well on optic nerve diameter. With the duplex mode arterial perfusion information of branches from the ophthalmic artery could be revealed. We recommend the method for neurological examination despite classical funduscopy.

\section{SESSION VII}

\section{Arterial wall dynamics \\ G.Baltgaile \\ Riga Stradina University, Neurological Department, Riga-Latvia}

An early change in arterial wall dynamics has been introduced as a novel risk factor for cardiovascular events in various populations.

Distensibility of an artery segment is a reflection of the mechanical stress affecting the arterial wall during the cardiac cycle. The stress can be defined as the difference in systolic and diastolic blood pressure and strain as the response of the artery system. This stress-strain relationship defined as distensibility has been investigated in the carotid arteries recent years thanks to the technological development of a high-resolution B-mode ultrasound.

$14^{\text {th }}$ ESNCH Meeting, Riga, Latvia, 2009 


\section{Cerebrovascular Diseases}

\section{$14^{\text {th }}$ ESNCH Meeting, Riga, Latvia, 2009}

A decrease of arterial distensibility (ie, increased artery wall stiffness) seems to be a common pathological mechanism for many factors associated with stroke arterial hypertension, diabetes mellitus, hyperlipidemia and myocardial infarction. Despite of common character of all parts of arterial wall dysfunction depended on many chemical and physical factors the measurements of arterial compliance, elastic modulus, augmentation pressure, stiffness and intimamedia thickness can indicate whether the changes in arterial distensibility mainly depends on endothelium dysfunction or perfusion pressure in association with aging, metabolic disorders and genetic factors.

The role of nervous regulation of blood vessel's tone in this process is not clear. Our previous and current studies show the strong correlation between dysfunction of autonomic nervous system and increase of carotid arterial distensibility in young patients. Paradoxically even hypertensive young patients have the same tendency despite of intima-media thickening correlated with hypertension.

The further development of measurements and analysis of arterial wall dynamics could help to define the mainly affected part of arterial wall involved in pathological process.

\section{Effect of risk factors on the morphology and function of arterial wall}

L. CSIBA, K. Csapó, S. Molnár, T. Magyar, L. Oláh

Department of Neurology, University of Debrecen, Hungary

Z. Bajkó, Cs.C. Szekeres, K.R. Kovács

Department of Neurology, Mures County Clinical Emergency

Hospital, Targu-Mures, Romania

Background: Clinical trials have indentified several factors that increase the risk of stroke. Most of these can be modified (high blood pressure, tobacco use, diabetes mellitus, high blood cholesterol etc) but some of them (age, gender, heredity, etc) can't be controlled. Our aim was to varify the effect of some controllable risk factors on the morphology and function of the arterial wall.

Methods: we examined 139 persons, 59 of them had recently diagnosed hypertension( $45.1 \pm 9.3$ years), 80 were normotensives (45.1 \pm 9.4 years). None of them suffered from diabetes and was smoker. The following examinations were performed: labs, measurement of the intima-media thickness (IMT) and arterial stiffness, head-up tilt table testing (HUTT) and neuropsychological tests.

Results: the persons were grouped as follows: controls (c), hyperlipidemic (L) (serum LDL-cholesterol $\geq 3.4 \mathrm{mmol} / \mathrm{L}$ ), hypertensives $(\mathrm{H})$, hypertensives with elevated lipids $(\mathrm{H}+\mathrm{L})$. IMT in the $C$ group: $0.54 \pm 0.08 \mathrm{~mm}<\mathrm{H}(0.62 \pm 0.1 \mathrm{~mm}, \mathrm{p}=0.01$ and $\mathrm{H}+\mathrm{L}(0.64 \pm 0.1 \mathrm{~mm}, \mathrm{p}=0.0006)$. By determining the stiffness parameters, significant differences were also observed in augmentation index and pulse wave velocity of the $C$ group compared with the $\mathrm{H}, \mathrm{H}+\mathrm{L}$ groups. Neuropsychological tests showed increased anxiety in the hypertensives. Results of the HUTT and of the examinations after 6 and 12 months of antihypertensive therapy will be also shown.

Conclusions: Morphological and functional impairments of arterial wall could be detected in the early stage of hypetension. These changes are more pronounced, when elevated serum lipids are also present. The early recognition and correct treatment of risk factors is important for preventing further impairments

\section{SESSION VIII}

\section{Detection of neurodegeneration \\ Uwe Walter, MD, PhD \\ University of Rostock, Department of Neurology \\ Gehlsheimer Str. 20, D-18147 Rostock, Germany}

Transcranial B-mode sonography (TCS) is a non-invasive, low-cost, short-duration neuroimaging method that allows high-resolution imaging of deep brain structures in patients with movement disorders. With contemporary high-end ultrasound systems, image resolution of echogenic deep brain structures can even be higher on TCS than on MRI, and neurodegenerative basal ganglia changes are often detected earlier with TCS than with MRI. On TCS, about $90 \%$ of patients with idiopathic Parkinson's disease (PD) exhibit abnormal hyperechogenicity of the substantia nigra (SN). This finding is already present in presymptomatic disease stages, suggesting TCS as a screening tool for populations at risk of later developing PD. SN hyperechogenicity discriminates PD from other Parkinsonian disorders such as multiple-system atrophy, progressive supranuclear palsy, vascular Parkinsonism and welding-related Parkinsonism. In turn, normal SN echogenicity in combination with lenticular nucleus hyperechogenicity indicates an atypical Parkinsonian syndrome rather than PD with a positive predictive value of more than $95 \%$. TCS shows characteristic basal ganglia changes also in other movement disorders such as hyperechogenic lesions of lenticular nucleus in idiopathic dystonia and in Wilson's disease, and of caudate nucleus in Huntington's disease. In multiple sclerosis, neurodegenerative disease-like basal ganglia changes can be frequently detected with TCS. TCS findings, especially of SN echogenicity, predict rate of further disease progression in MS. An emerging application of TCS is intra- and postoperative localization of deep brain stimulation electrodes in patients with movement disorders. 


\author{
Advanced technology for brain parenchyma imaging in \\ cerebrovascular disease \\ Rolf Kern, MD; \\ Department of Neurology, University Medical Centre Mannheim, \\ University of Heidelberg, Germany
}

In acute stroke, different sonographic methods can be used to assess structural and hemodynamic compromise. In particular, ultrasound perfusion imaging is a valuable approach for the non-invasive primary diagnosis and followup of cerebrovascular disease. Structural abnormalities of brain parenchyma such as intracerebral hemorrhage and complications (e.g. midline shift) can be detected by native transcranial multi-planar B-mode ultrasound. Advanced technology now allows improved depiction of cerebral structures through the human skull. Echo-contrast agents further increase the diagnostic confidence of brain parenchyma ultrasound imaging. One of the most important recent developments for cerebral contrast imaging is low mechanical index (MI) perfusion imaging, allowing for realtime assessment of microbubble bolus and refill kinetics. Coded contrast harmonics (CCH) using pulse compression provide highly sensitive contrast imaging with very low noise and longer contrast stability in the tissue than conventional imaging modes. Visualization of perfusion deficits in acute stroke patients can be significantly improved by microvascular imaging $(\mathrm{MVI})$ that tracks the passage of microbubbles through very small vessels after contrast bolus injection. MVI provides 'summation' images of microvascular flow in the parenchyma, and thus facilitates immediate stroke diagnosis under avoidance of time-consuming off-line analysis. 